\title{
Carência de Vitamina D numa População Hospitalar: Uma Fotografia pela Perspetiva Laboratorial
}

\author{
Vitamin D Insufficiency in a Hospital Population: A Photograph from the \\ Laboratory Perspective
}

\author{
Maria Joana SANTOS $\star^{* 1}$, Vera FERNANDES*1, Fernando Mota GARCIA ${ }^{2}$ \\ Acta Med Port 2015 Nov-Dec;28(6):726-734
}

\section{RESUMO}

Introdução: Apesar da hipovitaminose D ser cada vez mais reconhecida em todo o mundo, existem poucos estudos sobre a realidade portuguesa. Este trabalho pretende analisar o nível de vitamina D nos doseamentos realizados no nosso hospital e sua relação com idade, sexo, especialidade requisitante e momento da colheita.

Material e Métodos: Estudo observacional dos doseamentos de 25(OH)D realizados no nosso Hospital entre junho de 2012 e novembro de 2014. Variáveis estudadas: sexo, idade, especialidade requisitante, mês de colheita. O status de vitamina $D$ foi classificado como: 'Deficiência' ( $\leq 20 \mathrm{ng} / \mathrm{mL})$, 'Insuficiência' $(21-29 \mathrm{ng} / \mathrm{mL}$ ) e 'Suficiência' ( $\geq 30 \mathrm{ng} / \mathrm{mL}$ ).

Resultados: Incluímos 5439 doseamentos; $55,0 \%$ pertenciam a mulheres; a idade mediana foi 64,0 anos. Sessenta por cento apresentavam 'Deficiência', 20,7\% 'Insuficiência' e 18,9\% 'Suficiência'. Encontrámos uma correlação negativa entre idade e nível de vitamina $\mathrm{D}(p<0,001)$, não havendo diferenças significativas entre sexos. Nove especialidades requisitaram $98 \%$ dos doseamentos, destacando-se a Nefrologia $(56,2 \%)$. Encontrámos diferenças entre especialidades requisitantes relativamente à idade e nível de vitamina $\mathrm{D}(p<0,001)$. O nível de vitamina $\mathrm{D}$ variou ao longo do ano, com níveis superiores no verão, seguido do outono, primavera e inverno $(p<0,001)$. Apesar desta variação sazonal, a suficiência de vitamina $D$ foi sempre minoritária, sendo de $27,8 \%$ no Verão e $9,2 \%$ no Inverno.

Discussão: A carência de vitamina $D$ nesta população é elevada, transversal a todas as idades e não compensada pela variação sazonal da exposição solar.

Conclusão: A hipovitaminose D é um problema real, prevalente e merecedor de atuação na nossa população, atendendo às suas implicações clínicas.

Palavras-chave: Deficiência de Vitamina D; Estações do Ano; Portugal; Vitamina D.

\section{ABSTRACT}

Introduction: Although vitamin D deficiency is increasingly recognized around the world, there are few studies on the Portuguese reality. This study aims to analyse vitamin $D$ levels in the assays performed in our hospital and their relationship with age, genre, requesting specialty and moment of sample collection.

Material and Methods: Cross-sectional study of measurements of 25(HO)D performed in our Hospital between June 2012 and November 2014. Included variables: gender, age, requesting specialty, month of sample collection. Vitamin D status classified as: 'Deficiency' ( $\leq 20 \mathrm{ng} / \mathrm{mL}$ ), 'Insufficiency' ( $21-29 \mathrm{ng} / \mathrm{ml}$ ) and 'Sufficiency' ( $\geq 30 \mathrm{ng} / \mathrm{mL})$.

Results: We included 5439 assays; $55.0 \%$ from women; the median age was 64.0 years. Sixty per cent had 'Deficiency', $20.7 \%$ 'Insufficiency' and $18.9 \%$ 'Sufficiency'. We found a negative correlation between age and vitamin D level $(p<0.001)$. We didn't find differences in vitamin D levels between genres. Nine specialties requested $98 \%$ of the assays, namely Nephrology (56.2\%). We found differences between specialties based on age and vitamin D level $(p<0.001)$. Vitamin $\mathrm{D}$ levels changed throughout the year, with higher levels in the summer, followed by autumn, spring and winter $(p<0.001)$. Despite this seasonal fluctuation, vitamin $\mathrm{D}$ sufficiency was only present in a minority of assays ( $27.8 \%$ in summer and $9.2 \%$ in winter).

Discussion: Vitamin D deficiency is prevalent in this population, affects individuals of all ages and is not offset by the seasonal variation of sunlight.

Conclusion: Vitamin D deficiency is a real and prevalent problem in our population that needs further attention and action, given its clinical implications.

Keywords: Portugal; Seasons; Vitamin D; Vitamin D Deficiency.

\section{INTRODUÇÃO}

A vitamina $D$ é uma hormona lipossolúvel, obtida essencialmente a partir da alimentação e da síntese cutânea após a exposição solar. ${ }^{1}$ O 7-dehidrocolesterol, um derivado do colesterol e precursor da vitamina $D$ é produzido na pele por uma reação desencadeada pela radiação ultravioleta. Posteriormente, os metabolitos sintetizados são submetidos a duas reações de hidroxilação, a primeira no fígado originando a $25(\mathrm{OH}) \mathrm{D}$ e a segunda no rim formando a hormona metabolicamente ativa, 1,25(OH)2D ou calcitriol. ${ }^{1,2}$

As principais funções da $1,25(\mathrm{OH}) 2 \mathrm{D}$ no organismo relacionam-se com a regulação da homeostasia do cálcio e do fósforo, conjuntamente com a PTH. No intestino delgado, a vitamina $D$ é responsável pela absorção do cálcio e a nível renal contribui para reabsorção de cálcio e fósforo. A vitamina $D$ é ainda essencial para que ocorra a

\footnotetext{
* Co-primeiro autor.

1. Serviço de Endocrinologia. Hospital de Braga. Braga. Portugal.

2. Serviço de Patologia Clínica. Hospital de Braga. Braga. Portugal.

$\triangle$ Autor correspondente: Maria Joana Santos. mjoanasantos@hotmail.com.

Recebido: 29 de Janeiro de 2015 - Aceite: 08 de Julho de 2015 | Copyright @ Ordem dos Médicos 2015
} 
mineralização óssea. ${ }^{3}$

O status de vitamina $D$ é avaliado através do doseamento da $25(\mathrm{OH}) \mathrm{D}$, a sua principal forma circulante, com um tempo de semi-vida de duas a três semanas. ${ }^{4} \mathrm{~A}$ sua concentração reflete a disponibilidade de vitamina $D$ num período de quatro a seis semanas, o que a torna uma medida adequada das reservas da vitamina D. ${ }^{4,5}$

A 'deficiência' de vitamina $D$ define-se por um nível de 25(HO)D $\leq 20 \mathrm{ng} / \mathrm{mL}$; entre $21-29 \mathrm{ng} / \mathrm{mL}$ existe um estado de 'insuficiência' e níveis 30 - $100 \mathrm{ng} / \mathrm{mL}$ definem 'suficiência' de vitamina D. ${ }^{5}$ Apesar de não existir consenso sobre a concentração mínima de vitamina $D$ que garanta níveis ideais de saúde, não está demonstrado que níveis de vitamina $D$ superiores a $30 \mathrm{ng} / \mathrm{mL}$ se associem a maior benefício, havendo quem considere que níveis superiores a 50 $\mathrm{ng} / \mathrm{mL}$ possam estar associados a efeitos potencialmente adversos. ${ }^{5-7}$ Contudo, a intoxicação por vitamina $D$ é rara e geralmente ocorre com valores de vitamina $D>150 \mathrm{ng} /$ $\mathrm{mL} .{ }^{3,5}$

A carência de vitamina $D$ é prevalente em todo o mundo, pensando-se que atinja cerca de 1 bilião de indivíduos. ${ }^{6}$ Trata-se de um problema transversal a todas as idades nos países ocidentais, mas também na Ásia e Médio Oriente, e que tem vindo a aumentar. ${ }^{6,8-10}$ Mesmo em países tropicais as populações urbanas têm elevada prevalência de hipovitaminose D. ${ }^{4}$

Em Portugal, não existem estudos epidemiológicos acerca da prevalência de valores inadequados de vitamina D. Todavia, vários estudos europeus mostram proporções importantes de carência de vitamina $D$, sendo expectável que a realidade portuguesa seja semelhante. ${ }^{11}$

Assim, atendendo à prevalência significativa e crescente de carência de vitamina $\mathrm{D}$ e às suas implicações clínicas, este é considerado atualmente um importante problema de Saúde Pública. A identificação do problema é essencial dado que existe tratamento dirigido com suplementação farmacológica.

Este estudo teve como objetivos determinar o número de doseamentos de vitamina $D$ realizados no nosso hospital e a sua evolução recente; analisar o nível de vitamina D nos doseamentos realizados e estudar a relação entre o nível de vitamina $D$ e idade, sexo, especialidade requisitante e momento da colheita (estudo da variação sazonal).

\section{MATERIAL E MÉTODOS}

Realizámos um estudo observacional, em que procedemos ao levantamento de todos os doseamentos de $25(\mathrm{OH})$ D realizados no Serviço de Patologia Clínica do nosso Hospital entre 06 de junho de 2012 e 13 de novembro de 2014, relacionando-se este período com a disponibilidade de registos informatizados dos pedidos laboratoriais.

Para cada doseamento, foram avaliadas as seguintes variáveis: número do processo; sexo; idade; data da colheita e especialidade requisitante.

A 25(OH)D foi doseada por electroquimioluminescência no equipamento Liaison ${ }^{\circledR}$ (Diasorin) apresentando como valores de referência 30 a $100 \mathrm{ng} / \mathrm{mL}$. A amostra foi dividida em três grupos de acordo com os níveis séricos de $25(\mathrm{OH})$ $D$ : deficiência ( $\leq 20 \mathrm{ng} / \mathrm{mL})$, insuficiência $(21-29 \mathrm{ng} / \mathrm{mL})$ e suficiência ( $\geq 30 \mathrm{ng} / \mathrm{mL}){ }^{5}$

O número do processo clínico foi usado para identificar os doseamentos pertencentes aos mesmos indivíduos e calcular o número de doseamentos no mesmo doente.

A data da colheita foi organizada por meses, semestres e estações do ano. Considerou-se que os meses se distribuíam nas estações do ano da seguinte forma: inverno (janeiro a março); primavera (abril a junho); verão (julho a setembro); outono (outubro a dezembro).

Para a análise estatística recorreu-se ao programa estatístico IBM ${ }^{\circledR}$ SPSS $^{\circledR}$ Statistics v. 21. Para as variáveis contínuas apresentamos a mediana, percentis 25 e 75, valor mínimo e valor máximo. Para as variáveis categóricas apresentamos a frequência e percentagem. $\mathrm{Na}$ análise inferencial recorremos ao teste $\chi^{2}$ de Pearson para avaliar a associação entre duas variáveis qualitativas; à correlação de Pearson para avaliação de correlações entre variáveis quantitativas, e aos testes não-paramétricos Mann-Whitney e Kruskal-Wallis para comparação entre uma variável qualitativa e uma quantitativa, possuindo a última uma distribuição não normal. Considerou-se existir significância estatística para valores de $p<0,05$.

Durante todas as fases de elaboração do estudo foram respeitados os princípios éticos e deontológicos referentes à boa prática de recolha e análise estatística dos dados. O protocolo do trabalho de investigação foi aprovado pela Comissão de Ética do Hospital de Braga, de acordo com a declaração de Helsínquia.

\section{RESULTADOS \\ Caracterização da amostra}

No período de estudo, foram realizados 5456 doseamentos de vitamina $D$ no nosso hospital, provenientes de vários departamentos, nomeadamente: internamento, hospital de dia, consulta externa e serviço de urgência. Destes, 17 foram excluídos por terem sido realizados para o controlo de qualidade. Obteve-se uma amostra final constituída por 5439 doseamentos, correspondendo a 3257 doentes diferentes. A maioria dos doentes (2 016; 61,9\%) apresentava apenas um doseamento; 678 (20,8\%) tinham dois doseamentos; 331 (10,2\%) tinham três doseamentos; $149(4,6 \%)$ tinham quatro doseamentos. Os restantes 83 doentes $(2,5 \%)$ possuíam cinco ou mais determinações.

Dos 5439 doseamentos realizados, 2447 (45,0\%) correspondiam a indivíduos do sexo masculino e 2992 $(55,0 \%)$ a indivíduos do sexo feminino. A idade dos indivíduos apresentou uma mediana de 64,0 anos, com amplitude interquartis (AIQ): 46,0 - 76,0, variando de 0 a 99 anos. Verificou-se que $1,9 \%$ dos doseamentos foram feitos em doentes com idade até aos 19 anos; $13,9 \%$ entre os 20 - 39 anos; $26,5 \%$ entre os 40 - 59 anos e $57,6 \%$ em indivíduos a partir dos 60 anos de idade.

\section{Evolução do número de doseamentos}

No período estudado, o número de doseamentos de 
vitamina $D$ realizados aumentou cerca de 10 vezes, tendo sido feitos 35 pedidos no mês de junho de 2012 e 368 em outubro de 2014. O número de doseamentos por semestre aumentou progressivamente: junho a novembro de 2012: 259; dezembro de 2012 a maio de 2013: 736; junho a novembro de 2013: 1 195; dezembro 2013 a maio 2014: 1 555; junho a novembro de 2014: 1 694. O aumento percentual entre semestres consecutivos foi de $184,2 \%$, $62,4 \%, 30,1 \%$ e $8,9 \%$, respetivamente. O número de doseamentos realizados não variou significativamente ao longo das quatro estações do ano, tendo sido $20,9 \%$ realizados nos meses de inverno, $24,4 \%$ na primavera, $29,0 \%$ no verão e $25,6 \%$ no outono ( $p>0,05)$.

\section{Vitamina D}

O valor de vitamina $D$ apresentou uma distribuição não normal por enviesamento à esquerda, com uma mediana de 17,0, AIQ 10,0 - 26,0, variando entre 0 e $150 \mathrm{ng} / \mathrm{mL}$. A Fig. 1 mostra a distribuição do valor de vitamina $D$ nos doseamentos realizados. Relativamente ao status de vitamina D, $3281(60,3 \%)$ dos doseamentos correspondiam a deficiência, 1128 (20,7\%) a insuficiência e 1030 (18,9\%) a suficiência de vitamina $D$.

Encontrámos uma correlação negativa, estatisticamente significativa, entre a idade dos doentes e o valor absoluto de vitamina $D$, ou seja, quanto maior a idade dos doentes, menor o valor de vitamina $D(p<0,001 ; r=-0,054)$. Da mesma forma, o status de vitamina $D$ também foi diferente de acordo com a idade ( $p<0,001)$ (Fig. 2).

O nível de vitamina $D$ não foi significativamente diferente entre homens e mulheres (Med 17,0, AIQ 11,0 - 27,0 vs Med 17,0, AIQ 10,0 - 26,0) ( $p>0,05)$. Da mesma forma, a distribuição entre homens/mulheres de deficiência (44,4\% I $55,6 \%)$, insuficiência $(44,6 \% / 55,4 \%)$ e suficiência $(47,4 \%$ / 52,6\%) de vitamina D não foi diferente $(p>0,05)$.

\section{Especialidades requisitantes}

Relativamente às especialidades que requisitaram os doseamentos de vitamina $D$, verificámos que nove especialidades foram responsáveis por $98 \%$ dos pedidos. A distribuição por especialidades foi a seguinte: Nefrologia (3 045; $56,2 \%)$; Endocrinologia (439; 8,1\%); Medicina Interna (436; $8,0 \%)$; Cirurgia Geral (429; 7,9\%); Gastrenterologia (324; $6,0 \%)$; Reumatologia (264; 4,9\%); Consulta Multidisciplinar de Obesidade (213; 3,9\%); Neurologia (104; 1,9\%); Pediatria $(58 ; 1,1 \%)$; outras especialidades $(108 ; 2,0 \%)$.

Encontrámos diferenças significativas entre especialidades relativamente à idade e ao nível de vitamina $\mathrm{D}$ (Tabela 1).

Da análise estatística entre especialidades relativamente à idade, excluíram-se as categorias 'Pediatria' e 'Outras'. Entre as restantes, encontraram-se diferenças estatisticamente significativas entre todas as especialidades $(p<0,05)$ exceto entre Consulta Multidisciplinar de Obesidade (CMO), Gastrenterologia, Cirurgia Geral e Neurologia; entre Medicina Interna e Nefrologia. Assim, as especialidades de Medicina Interna e Nefrologia apresentaram uma idade mediana significativamente superior às outras especialidades, enquanto as especialidades de Gastro, Cirurgia Geral, Neurologia e CMO foram as que apresentaram doentes mais jovens $(p<0,05)$.

Excluindo a categoria 'outras' da comparação entre especialidades, dado ser uma categoria de composição heterogénea, verificou-se que existiam diferenças estatisticamente significativas no nível de vitamina $D$ entre as seguintes especialidades: Medicina Interna versus todas exceto Neurologia e CMO; CMO versus todas exceto Medicina Interna, Neurologia e Gastrenterologia; Neurologia, Gastrenterologia, Nefrologia e Cirurgia Geral versus Endocrinologia, Reumatologia e Pediatria. Assim, pode concluir-se que a especialidade de Medicina Interna apresentou

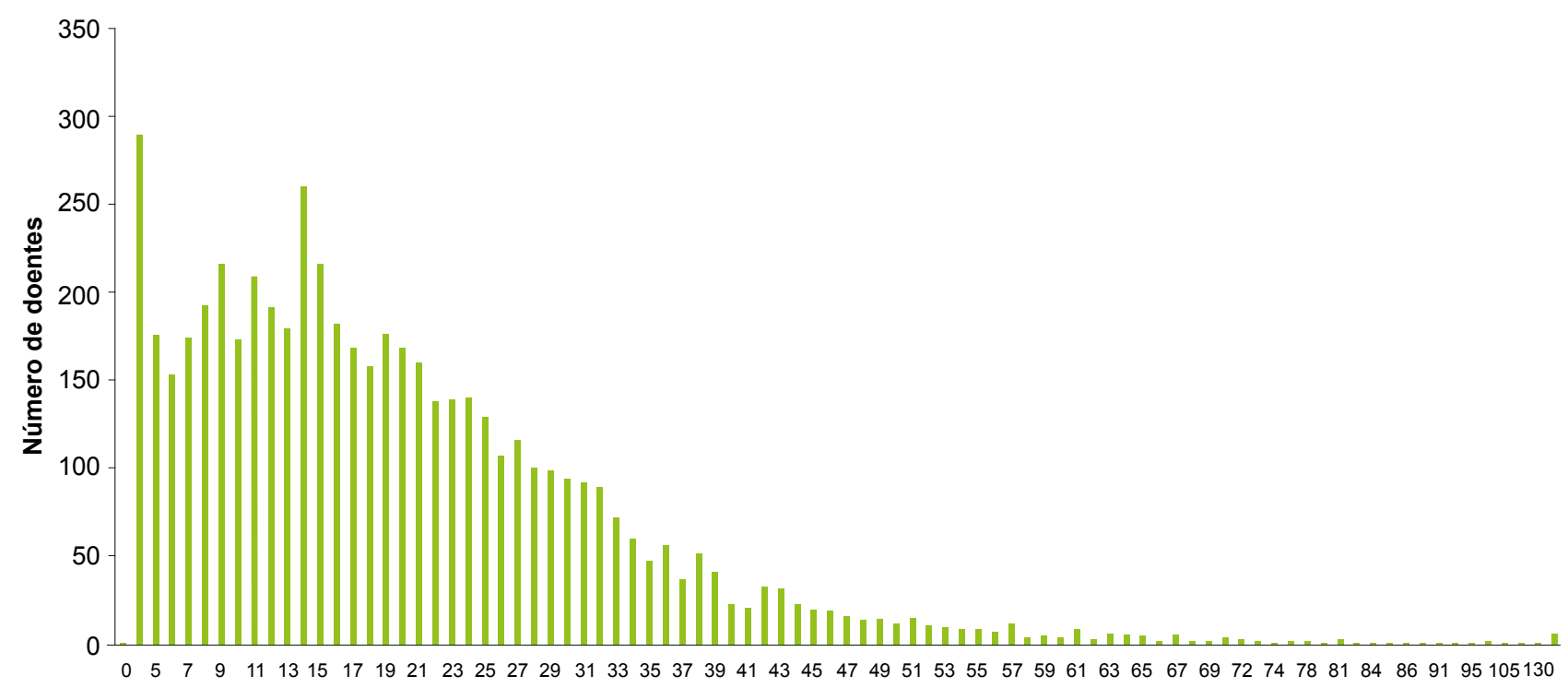

Vitamina $D(\mathrm{ng} / \mathrm{mL})$

Figura 1 - Valor de vitamina $D(\mathrm{ng} / \mathrm{mL})$ nos doseamentos efetuados 


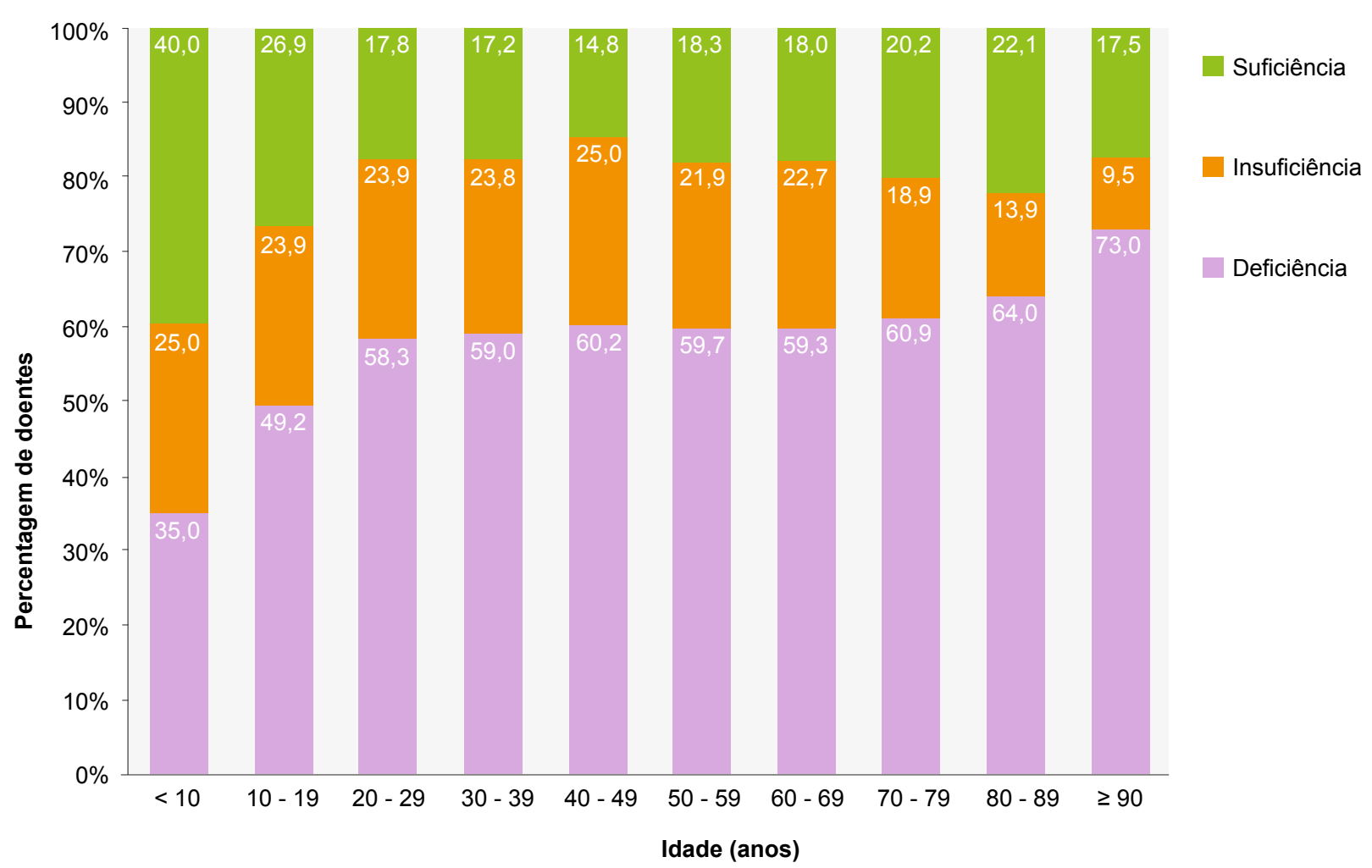

Figura 2 - Distribuição do status de vitamina D por grupo etário

Tabela 1 - Distribuição da idade (anos) e níveis de vitamina $D(\mathrm{ng} / \mathrm{mL})$ por especialidade requisitante

\begin{tabular}{|c|c|c|c|c|c|c|c|c|}
\hline & \multicolumn{4}{|c|}{$\begin{array}{c}\text { Vitamina D } \\
(\mathrm{ng} / \mathrm{mL})\end{array}$} & \multicolumn{4}{|c|}{$\begin{array}{l}\text { Idade } \\
\text { (anos) }\end{array}$} \\
\hline & $\begin{array}{c}\text { Med } \\
\text { (P25 - P75) }\end{array}$ & Min & $\operatorname{Max}$ & $p$ & $\begin{array}{c}\text { Med } \\
\text { (P25 - P75) }\end{array}$ & Min & Max & $p$ \\
\hline Cirurgia Geral & $\begin{array}{c}18,0 \\
(12,0-24,0)\end{array}$ & 4,0 & 67,0 & $<0,001$ & $\begin{array}{c}44,0 \\
(37,0-51,0)\end{array}$ & 19,0 & 82,0 & $<0,001$ \\
\hline Gastrenterologia & $\begin{array}{c}16,0 \\
(11,0-22,0)\end{array}$ & 4,0 & 81,0 & & $\begin{array}{c}43,0 \\
(32,0-56,0)\end{array}$ & 17,0 & 86,0 & \\
\hline Medicina Interna & $\begin{array}{c}12,0 \\
(6,25-21,0)\end{array}$ & 0,0 & 102,0 & & $\begin{array}{c}71,0 \\
(55,0-81,0)\end{array}$ & 18,0 & 99,0 & \\
\hline Nefrologia & $\begin{array}{c}17,0 \\
(10,0-27,0)\end{array}$ & 4,0 & 150,0 & & $\begin{array}{c}71,0 \\
(60,0-79,0)\end{array}$ & 18,0 & 95,0 & \\
\hline $\begin{array}{l}\text { C. Multidisciplinar } \\
\text { Obesidade }\end{array}$ & $\begin{array}{c}15,0 \\
(10,0-20,0)\end{array}$ & 4,0 & 50,0 & & $\begin{array}{c}40,0 \\
(33,0-49,0)\end{array}$ & 17,0 & 68,0 & \\
\hline Neurologia & $\begin{array}{c}14,0 \\
(9,25-24,75)\end{array}$ & 4,0 & 150,0 & & $\begin{array}{c}42,0 \\
(33,25-53,75)\end{array}$ & 7,0 & 85,0 & \\
\hline Endocrinologia & $\begin{array}{c}21,0 \\
(14,0-30,0)\end{array}$ & 4,0 & 102,0 & & $\begin{array}{c}54,0 \\
(42,0-65,0)\end{array}$ & 13,0 & 88,0 & \\
\hline Reumatologia & $\begin{array}{c}20,0 \\
(13,0-30,0)\end{array}$ & 4,0 & 95,0 & & $\begin{array}{c}62,0 \\
(56,0-70,75)\end{array}$ & 17,0 & 94,0 & \\
\hline Pediatria & $\begin{array}{c}25,0 \\
(16,75-31,25)\end{array}$ & 4,0 & 57,0 & & $\begin{array}{c}8,5 \\
(1,75-14,0)\end{array}$ & 0,0 & 17,0 & \\
\hline Outras & $\begin{array}{c}21,0 \\
(12,2-29,0)\end{array}$ & 4,0 & 150,0 & & $\begin{array}{c}54,0 \\
(37,25-67,0)\end{array}$ & 0,0 & 87,0 & \\
\hline
\end{tabular}


valores de vitamina $D$ significativamente inferiores a quase todas as outras especialidades e que a Endocrinologia, Reumatologia e Pediatria tinham valores significativamente superiores relativamente às restantes especialidades $(p<$ $0,05)$. A Fig. 3 mostra a distribuição significativamente diferente das três categorias de status de vitamina $D$ entre as várias especialidades $(p<0,001)$.

\section{Estação do ano}

O nível de vitamina $D$ variou de forma significativa entre as quatro estações do ano $(p<0,001)$. Os níveis mais elevados verificaram-se nos meses de verão (Med 22,0; AIQ $14,0-31,0 \mathrm{ng} / \mathrm{mL}$ ), seguidos dos meses de outono (Med 18,0; AIQ 11,0-27,0 ng/mL), primavera (Med 16,0; AIQ 10,0 - 25,0 ng/mL) e inverno (Med 13,0; AIQ 7,0 - 20,0 ng/mL).

Quando analisada a variação nos 12 meses do ano, verificámos que, mesmo dentro de cada estação, havia uma variação progressiva, tal como demonstrado na Fig. 4. O mês com menor nível de vitamina $D$ foi março (Med 12,0; AIQ 6,5 - 21,0 $\mathrm{ng} / \mathrm{mL}$ ), correspondendo ao final do inverno e o mês com nível mais elevado foi setembro (Med 23,0; AIQ 14,0 - 31,0 ng/mL), coincidindo com o final do verão. No entanto, mesmo nos meses com níveis mais elevados de vitamina $D$, estes mantiveram-se baixos, com medianas inferiores ao limite que define a suficiência.

De forma concordante, o status de vitamina D acompanhou a variação sazonal dos níveis absolutos de vitamina D (Fig. 5). Esta análise permitiu verificar que existem diferenças estatisticamente significativas no valor de vitamina $D$ nas quatro estações do ano ( $p<0,001$ em todas as comparações entre estações). Os meses de verão foram os que apresentaram valores mais elevados de vitamina $D$, seguindo-se os meses de outono, primavera e por fim inverno. Contudo, verificámos que apesar da melhoria dos níveis de vitamina $D$ nos meses de maior exposição solar, a percentagem de doentes com suficiência variou pouco nas 4 estações e foi sempre minoritária. Nos doentes com suficiência de vitamina $D$, o valor mediano desta vitamina não variou significativamente entre as quatro estações (inverno: Med 36,0; AIQ 32,0 - 42,5 ng/mL; primavera: Med 37,0; AIQ 32,0 - 46,0 ng/mL; verão: Med 37,5; AIQ 32,0 - 45,0 $\mathrm{ng} / \mathrm{mL}$; outono: Med 36,0; AIQ 32,0 - 44,0 ng/mL) $(p>0,05)$.

\section{DISCUSSÃO}

No período analisado verificámos um aumento progressivo dos doseamentos de Vitamina D no nosso hospital, que poderá ter várias explicações. A carência de vitamina $\mathrm{D}$ tem sido potencialmente associada a várias patologias, o que aumentou o interesse da comunidade científica neste tema. Consequentemente, tem havido um aumento no número de publicações científicas relacionadas com este tema, assim como dos doseamentos realizados e dos gastos em suplementos desta vitamina em vários países. ${ }^{10} \mathrm{Por}$ outro lado, no período estudado, houve um crescimento significativo do corpo clínico da Nefrologia no nosso hospital. Dada a estreita relação da insuficiência renal crónica com a carência de vitamina $D$, o aumento do número destes doseamentos era expectável.

Verificámos que $97,5 \%$ dos doentes tiveram no máximo quatro doseamentos da vitamina $\mathrm{D}$. Dado que, em indivíduos com deficiência, está preconizada a monitorização 3 - 4 meses após o início do tratamento e, atendendo à

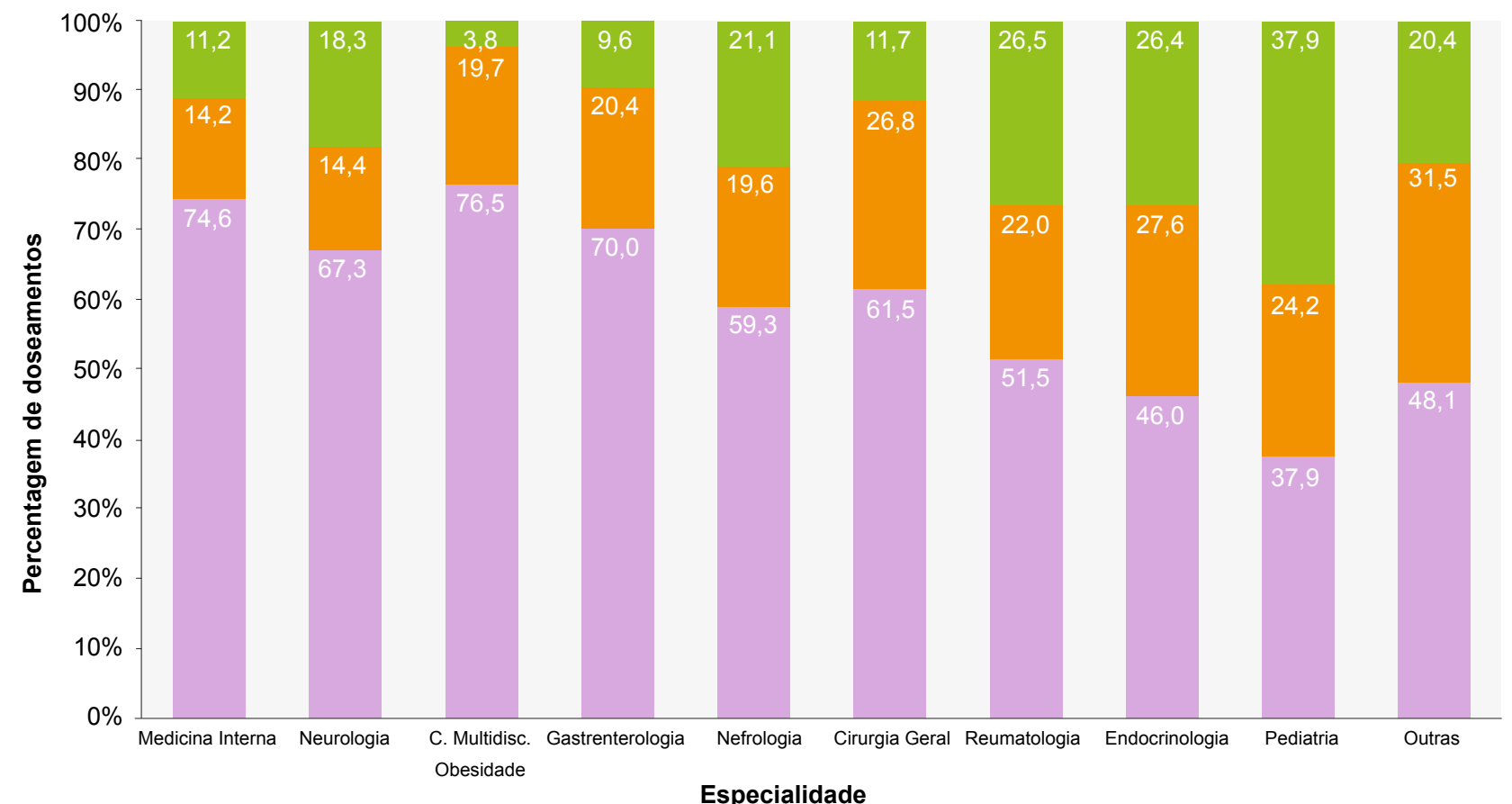

Suficiência

- Insuficiência

Deficiência

Figura 3 - Distribuição do status de vitamina D por especialidade requisitante 


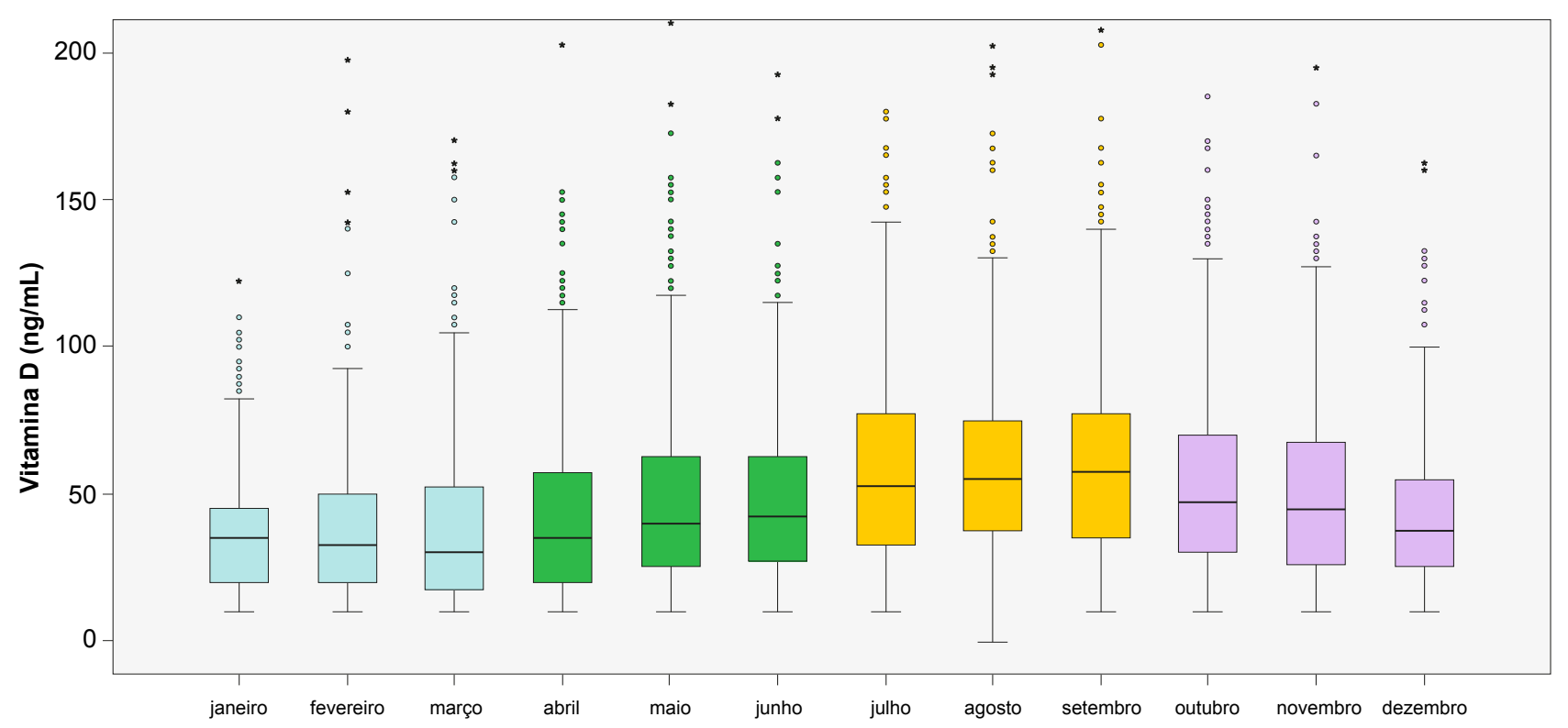

Mês de colheita

Figura 4 - Variação dos níveis de vitamina $\mathrm{D}(\mathrm{ng} / \mathrm{mL})$ ao longo dos 12 meses

elevada proporção de doentes com níveis inadequados, pode considerar-se um resultado expectável. ${ }^{12}$

Neste trabalho, encontrámos uma elevada carência de vitamina $\mathrm{D}$, fato concordante com outros estudos que mostraram que se trata de um problema transversal a muitos países e a todas as faixas etárias. ${ }^{5}$ Nos EUA, em 2005 2006, o nível médio de $25(\mathrm{OH}) \mathrm{D}$ da população era de 19,9 $\mathrm{ng} / \mathrm{mL}$, havendo deficiência em $63 \%$ dos hispânicos e $82 \%$ dos indivíduos de raça negra. ${ }^{10}$ Mesmo em países com elevada exposição solar, estima-se que 30 - 50\% da população tenha deficiência de vitamina D. ${ }^{6}$ Não existem estudos populacionais dos níveis de vitamina $\mathrm{D}$ em Portugal, apenas estudos limitados a populações específicas. Um trabalho com doentes internados num Serviço de Medicina Interna encontrou $92,7 \%$ de deficiência. ${ }^{13}$ Esta prevalência muito elevada, poder-se-á dever ao fato de se tratar de um estudo limitado aos meses de abril-maio, apenas com doentes idosos, internados, com índice funcional elevado, polimedicação e múltiplas comorbilidades. Outro estudo em doentes internados por fratura de fragilidade do punho, entre junho - outubro, medicados e não medicados com suplementação vitamínica, mostrou suficiência em $26,2 \%$, insuficiência em $45,2 \%$ e deficiência em $28,6 \% .{ }^{14}$ Um estudo português mais recente, semelhante ao nosso, encontrou níveis de insuficiência e deficiência semelhantes aos que descrevemos. Incluiu 2071 doseamentos de vitamina D entre setembro - dezembro de 2010: $88,1 \%$ apresentaram níveis $<30 \mathrm{ng} / \mathrm{mL}$ e $65 \%<20 \mathrm{ng} / \mathrm{mL} .{ }^{15}$

A elevada carência de vitamina $D$ no século $X X I$ deve-se, essencialmente, à diminuição da síntese cutânea de vitamina $\mathrm{D}$, associada ao estilo de vida das populações urbanas. A maior fonte de vitamina $D$ para os humanos é a exposição à luz solar (influenciada pela latitude, tempo de exposição, hora do dia e estação do ano), que está franca- mente diminuída, uma vez que a maioria dos indivíduos realiza grande parte das atividades em espaços fechados..$^{4,5} \mathrm{O}$ uso de protetores solares, que diminuem significativamente a absorção de raios UVB (redução $\geq 95 \%$ com o fator de proteção 30 ) também contribui para este fenómeno. 5,6 A diminuição da biodisponibilidade de vitamina $D$, existente nas síndromes de malabsorção de gorduras, obesidade, alimentação pobre em alimentos ricos/suplementados em vitamina $D$ e amamentação exclusiva, também causa carência de vitamina D. ${ }^{5,6} \mathrm{Na}$ Europa e em Portugal, onde poucos alimentos são suplementados com vitamina $D$, este risco é maior. ${ }^{5,6}$ Numa amostra de mulheres adultas residentes no Porto, verificou-se que a ingestão inadequada de vitamina $D$ era de $70,5 \%$ em mulheres com menos de 30 anos e $96,0 \%$ em mulheres dos 60 - 69 anos, aumentando com a idade. ${ }^{16}$ Por outro lado, o metabolismo aumentado da vitamina $D$ provocado por alguns fármacos (antiepilépticos, antiretrovirais, glucocorticóides) ou doenças granulomatosas crónicas (tuberculose, sarcoidose), assim como a diminuição da síntese de 25(OH)D (insuficiência hepática severa), o aumento da perda de $25(\mathrm{OH}) \mathrm{D}$ (síndrome nefrótica) e a diminuição da síntese de 1,25(OH)2D (doença renal crónica estádios 4 e 5) são também causas de carência de vitamina D. 5,6

$\mathrm{Na}$ nossa amostra, alguns doentes apresentavam valores de vitamina D superiores a $100 \mathrm{ng} / \mathrm{mL}$. Vários estudos demonstraram que níveis muito elevados de vitamina $\mathrm{D}$ não trazem benefícios adicionais e estão associados a doença cardiovascular, quedas, fragilidade, carcinoma do pâncreas e aumento de mortalidade global. ${ }^{7}$ Os níveis de vitamina $D$ devem ser mantidos $<100 \mathrm{ng} / \mathrm{mL}$, uma vez que para valores $>150 \mathrm{ng} / \mathrm{mL}$ existe risco de hipercalcemia. ${ }^{5}$

$\mathrm{Na}$ nossa amostra, verificámos que os indivíduos com mais idade tinham níveis mais baixos de vitamina $D$. Vários 


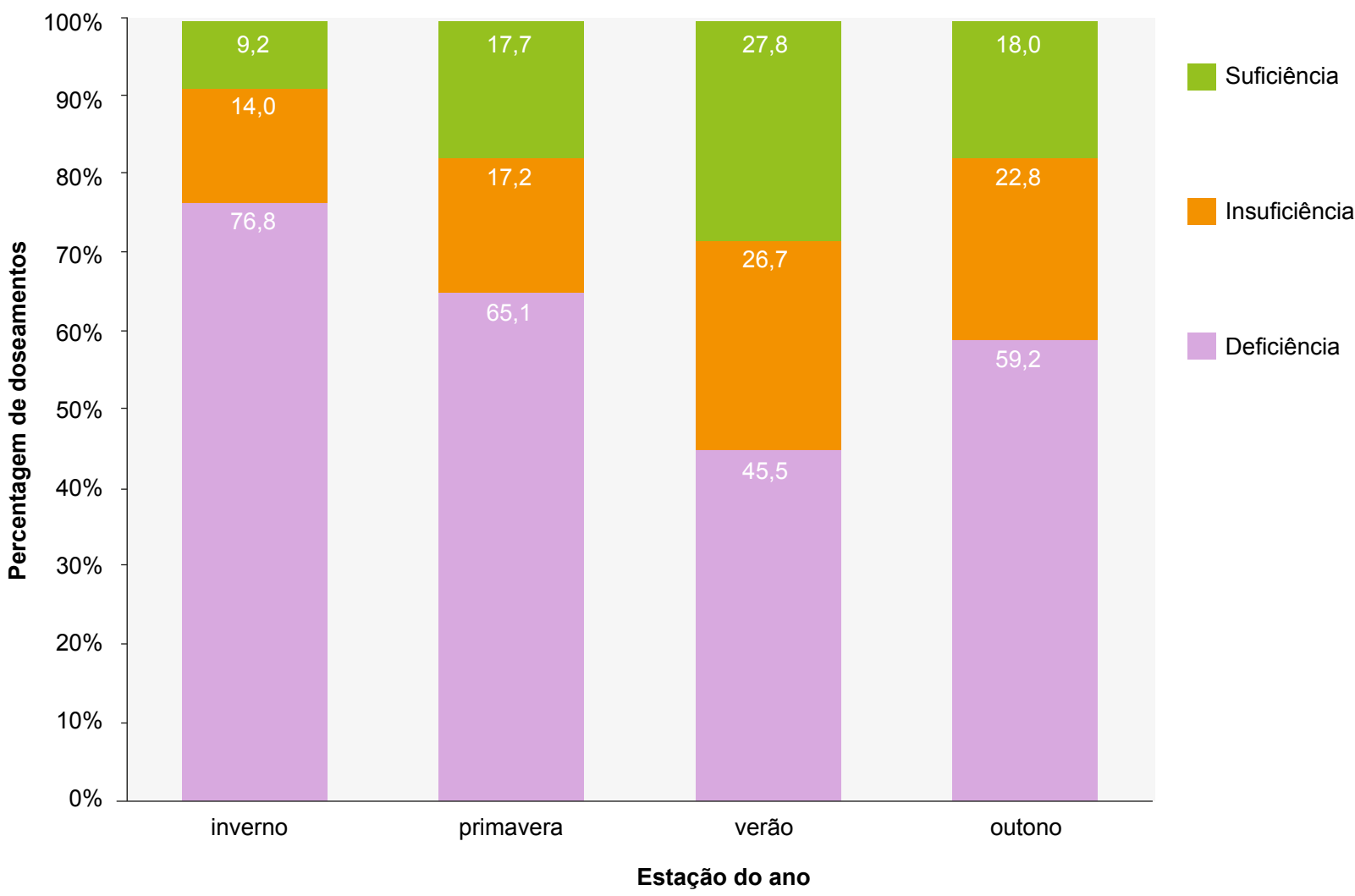

Figura 5 - Distribuição do status de vitamina D por estação do ano

estudos confirmam a elevada prevalência de deficiência de vitamina $\mathrm{D}$ em idosos. Um estudo europeu nos anos 80 mostrou que $36 \%$ dos homens e $47 \%$ das mulheres idosos tinham deficiência de vitamina $D$, que era mais grave nos países do sul da Europa. ${ }^{4}$ Estudos mais recentes apontam para uma prevalência de deficiência de vitamina $D$ nos idosos americanos e europeus que vivem na comunidade entre $40-100 \%{ }^{5.6,9}$ Apesar da carência de vitamina D ser transversal a todas as idades, os idosos são uma população de maior risco, devido à menor realização de atividades ao ar livre, ingestão alimentar, absorção intestinal e síntese cutânea de vitamina D.,5,9 Nos indivíduos institucionalizados este risco é ainda maior. ${ }^{17}$ Vários estudos observacionais mostraram existir associação entre os níveis séricos de vitamina $D$ (nomeadamente $<20 \mathrm{ng} / \mathrm{mL}$ ) e a propensão para quedas e fraturas. ${ }^{3,17} \mathrm{O}$ défice de vitamina D nos idosos é um problema potencialmente mais grave, que pode pôr em risco a independência funcional destes doentes. ${ }^{4}$

Verificámos que, apesar dos níveis de vitamina $D$ serem melhores nos indivíduos em idade pediátrica, a suficiência nesta faixa etária era de apenas $37,9 \%$. Estudos internacionais e nacionais corroboram a baixa prevalência de suficiência de vitamina $D$ nos jovens. Um estudo americano demonstrou que a prevalência de deficiência de vitamina $D$ era de $48 \%$ em pré-adolescentes de raça branca do sexo feminino. ${ }^{5} \mathrm{Na}$ Alemanha, num estudo nacional com mais de 10000 crianças e adolescentes, esta prevalência foi de $87 \% .^{9}$ Dois trabalhos portugueses mostraram resultados pouco animadores. Num estudo com 122 crianças entre os
5 - 18 anos, 92,5\% tinham insuficiência de vitamina $D$, dos quais $>40 \%$ tinham deficiência. ${ }^{18} \mathrm{Um}$ outro com 73 crianças, revelou níveis de suficiência de vitamina $D$ em $<20 \%$, tendo $30,9 \%$ défice e $42,5 \%$ insuficiência. Este último estudo confirmou também que as crianças em idade escolar tinham níveis piores de vitamina $D$ do que as crianças mais pequenas. ${ }^{19}$

Não encontrámos diferenças significativas entre sexos, tal como esperado, dado que a síntese de vitamina $D$ não se relaciona com esta variável e tal como descrito noutros trabalhos. ${ }^{13}$

O rastreio da carência de vitamina $D$ não está recomendado na população em geral e deve ser reservado para as situações de elevado risco de deficiência. ${ }^{5}$ Neste trabalho, verificámos que as nove especialidades mais requisitantes de doseamento de $21(\mathrm{OH}) \mathrm{D}$ representam os principais subgrupos de doentes com maior risco para deficiência de vitamina D. São exemplos disso a Nefrologia, Endocrinologia, Gastrenterologia e a CMO. ${ }^{6}$

Como seria de esperar, encontrámos diferenças estatisticamente significativas na idade dos doentes entre as diferentes especialidades. A Medicina Interna e Nefrologia, reflexo da patologia mais prevalente nestas especialidades, apresentaram os doentes mais idosos.

Encontrámos diferenças significativas nos níveis de vitamina D entre as várias especialidades. A Medicina Interna apresentou níveis significativamente menores de vitamina $D$, consequência provável da maior idade dos doentes. No entanto, tal não se verificou na Nefrologia, cujos doentes, apesar da idade, tinham um status de vitamina $D$ melhor, 
podendo traduzir um maior tratamento desta hipovitaminose. A Pediatria, Endocrinologia e Reumatologia obtiveram os melhores resultados. Na Pediatria, este dado provavelmente resulta da menor idade dos doentes e de uma atitude mais ativa no tratamento. A Endocrinologia e a Reumatologia são especialidades em que é natural existir uma preocupação especial com este problema, devido às suas consequências esqueléticas/metabólicas. A Nefrologia será também de englobar nesta categoria, podendo eventualmente justificar-se os seus piores resultados por esta especialidade ser recente no nosso hospital. As restantes especialidades tiveram um comportamento intermédio, com níveis de vitamina $D$ aquém do desejado, nomeadamente em populações de risco para esta hipovitaminose. No caso da Consulta Multidisciplinar de Obesidade, é sabido que existe uma relação inversa entre IMC $\geq 30 \mathrm{Kg} / \mathrm{m}^{2}$ e níveis séricos de 25(HO)D. ${ }^{5}$ Doentes obesos têm, geralmente, níveis de vitamina $D$ entre os $10-20 \mathrm{ng} / \mathrm{mL}$, provavelmente não só pelo sequestro desta vitamina no tecido adiposo, mas também por menores níveis de exercício físico e exposição solar do que indivíduos normoponderais. ${ }^{3}$ Apesar destas diferenças entre especialidades, os níveis de vitamina $D$ encontrados foram globalmente baixos e longe do valor ideal. ${ }^{6}$

Apesar de não existir variação significativa na data de colheita, verificámos que existiam diferenças significativas nos níveis de $25(\mathrm{OH}) \mathrm{D}$ entre as estações do ano, com níveis mais elevados no verão, seguindo-se o outono, a primavera e o inverno. Os níveis do outono refletem provavelmente a exposição solar do verão, dado que os níveis sérios de $25(\mathrm{OH}) \mathrm{D}$ são máximos cerca de 30 - 60 dias após o pico de exposição solar nos meses de verão. ${ }^{20}$ Curiosamente, mesmo em cada estação, existia uma evolução progressiva nos níveis de vitamina $D$ de acordo com a intensidade sazonal da luz solar. É sabido que, no hemisfério norte, a variação sazonal da luz solar é de cerca de $20 \%$ do verão para o inverno e que a luz mais eficaz na síntese de vitamina $D$ ocorre entre as $10 \mathrm{~h}$ e $15 \mathrm{~h}$ na primavera, verão e outono, tornando a relação risco/benefício entre o risco de cancro de pele e produção de vitamina $\mathrm{D}$ um problema em debate. ${ }^{5,21}$

Em todas as estações, a mediana de vitamina $D$ foi inferior ao limite para a sua suficiência, mesmo tratando-se um país com elevada exposição solar. Como já descrito, são vários os fatores associados ao nosso estilo de vida que o podem justificar. Verificámos um aumento de 69,2\% nos doentes com deficiência de vitamina $D$ do verão para o inverno. A exposição solar adequada nos meses de maior intensidade solar pode permitir obter níveis adequados de vitamina $D$, que são armazenados no tecido adiposo e libertados durante o inverno, altura em que a síntese de vitamina $D$ é menos eficaz. ${ }^{6}$ Contudo, colocámos a hipótese de que os doentes com suficiência de vitamina $D$ nos meses de maior exposição solar, apresentassem níveis limítrofes de suficiência, não tendo reservas capazes de manter os níveis de vitamina $D$ nos meses de menor exposição solar. De fato, verificámos que o nível de vitamina $D$ dos doentes com suficiência nas diferentes estações do ano não variava significativamente e que a sua mediana se encontrava no limite da normalidade. Assim, conclui-se que mesmo os doentes com suficiência de vitamina $D$ apresentavam reservas baixas, o que diminuiu a disponibilidade da vitamina para os meses de menor exposição solar.

\section{Pontos fortes e limitações}

Este trabalho, que incluiu um elevado número de doseamentos de vitamina $D$ num período de estudo alargado, permitiu conhecer melhor o status de vitamina $D$ dos doentes seguidos no nosso hospital. Todavia, o fato de ter sido realizado num único hospital impossibilita a generalização dos resultados. Além disso, apresenta as limitações inerentes a um estudo observacional e retrospetivo. Por fim, a ausência de dados relativos a variáveis que estão relacionados com o status de vitamina $D$, nomeadamente raça/ etnia, comorbilidades, índice funcional, hábitos dietéticos e estado nutricional, número de horas de exposição solar por dia, índice de massa corporal, tratamento de substituição atual ou recente e medicação habitual, não permitiu explicar com mais certeza alguns dos dados encontrados.

\section{CONCLUSÃO}

Com este trabalho demonstrámos que, nos doseamentos recentes de vitamina $D$ no nosso hospital, a carência de vitamina $D$ é muito prevalente. Apesar dos seus níveis variarem significativamente nas diferentes estações do ano, em estreita relação com o número de horas e intensidade solar sazonal, ficaram sempre aquém do desejado, mesmo no verão. As reservas nos doentes com suficiência parecem ser diminutas, atendendo ao aumento da proporção de doentes com deficiência no inverno. Consideramos que estes resultados revelam um estado de carência de vitamina $D$ merecedor de atuação, atendendo às implicações clínicas desta hipovitaminose. Esperamos que o reconhecimento desta situação possibilite a melhoria dos cuidados futuramente prestados aos nossos doentes.

\section{PROTECÇÃO DE PESSOAS E ANIMAIS}

Os autores declaram que o protocolo do trabalho de investigação foi aprovado pela Comissão de Ética do Hospital de Braga, de acordo com a declaração de Helsínquia.

\section{CONFIDENCIALIDADE DOS DADOS}

Os autores declaram ter seguido os protocolos do seu centro de trabalho acerca da publicação dos dados de doentes.

\section{CONFLITOS DE INTERESSES}

Os autores declaram não ter nenhum conflito de interesses relativamente ao presente artigo.

\section{FONTES DE FINANCIAMENTO}

Não existiram fontes externas de financiamento para a realização deste artigo. 


\section{REFERÊNCIAS}

1. Bringhurst FR, Demay MB, Kronenberg HM. Hormones and disorders of mineral metabolism. In: Melmed S, Polonsky KS, Larsen PR, Kronenberg $\mathrm{HM}$, editors. Williams textbook of endocrinology. Philadelphia: Elsevier Saunders; 2011. p. 1237-304.

2. Shoback D, Sellmaeyer D, Bikle DD. Metabolic bone disease. In: Gardne DG, Shoback D, editors. Greenspan's basic \& clinical endocrinology. New York: The McGraw-Hill; 2011. p. 227-84.

3. Rosen CJ. Vitamin D insufficiency. N Eng J Med. 2011;364:248-54

4. Boucher BJ. The problems of vitamin $D$ insufficiency in older people. Aging Dis. 2012;3:313-29.

5. Holick MF, Binkley NC, Bischoff-Ferrari HA, Gordon CM, Hanley DA, Heaney RP, et al. Evaluation, treatment, and prevention of vitamin D deficiency: an Endocrine Society Clinical Practice Guideline. J Clin Endocrinol Metab. 2011;96:1911-30.

6. Holick MF. Vitamin D deficiency. N Eng J Med. 2007;357:266-81.

7. Ross AC, Manson JE, Abrams SA, Aloia JF, Brannon PM, Clinton SK, et al. The 2011 report on dietary reference intakes for calcium and vitamin D from the Institute of Medicine: what clinicians need to know. J Clin Endocrinol Metab. 2011;96:53-8.

8. Girgis CM, Clifton-Bligh RJ, Hamrick MW, Holick MF, Gunton JE. The roles of vitamin $D$ in skeletal muscle: form, function, and metabolism. Endocr Rev. 2013;34:33-83.

9. Domarus CV, Dipl-Ing JB, Barvencik F, Amling M, Pogoda P. How much vitamin $D$ do we need for skeletal health? Clin Orthop Relat Res. 2011;469:3127-33.

10. Engelman CD. Vitamin D recommendations: the saga continues. J Clin Endocrinol Metab. 2011;96:3065-6.

11. Pisco L, Barros $H$, Mascarenhas $M$, Carvalheiro $M$, Cantista $P$, Laíns J, et al. Declaração Portuguesa da Vitamina D. 2009; [consultado 2015 jan 02]. Disponível em: http://www.spmi.pt/pdf/Declaracao_Port VitD_2009_final.pdf.

12. Dawson-Hughes B. Vitamin D deficiency in adults: Definition, clinical manifestations, and treatment. Uptodate. 2014; [consultado 2015 jan 03]. Disponível em: http://www.uptodate.com/contents/vitamin-ddeficiency-in-adults-definition-clinical-manifestations-and-treatment? source=machineLearning \&search=deficency+vitamin $+d \&$ selectedTit le=1 150\&sectionRank=1\&anchor=H7\#H2.

13. Santiago T, Rebelo M, Porto J, Silva N, Vieira J, Nascimento Costa JM Hipovitaminose D em doentes internados num Serviço de Medicina Interna. Acta Med Port. 2012;25:68-76.

14. Silva L, Freitas J, Sampaio L, Terroso G, Pinto JM, Veludo V, et al. Níveis séricos de vitamina $D$ em portugueses com fracturas de fragilidade. Acta Reumatol Port. 2010;35:352-7.

15. Alves M, Bastos M, Leitão F, Marques G, Ribeiro G, Carrilho F. Vitamina D - importância da avaliação laboratorial. Rev Port Endocrinol Diabetes Metab. 2013;8:32-9.

16. Lucas $R$, Costa $L$, Barros $H$. Ingestão de cálcio e vitamina $D$ numa amostra urbana de mulheres Portuguesas. Arq Med. 2005;19:7-14.

17. Rosen CJ, Adams JS, Bikle DD, Black DM, Demay MB, Manson JE, et al. The nonskeletal effects of vitamin D: an Endocrine Society scientific statement. Endocr Rev. 2012;33:456-92.

18. Ferreira S, Nascimento S, Barros C, Tomada I, Carreiro E, Rêgo C. Status de vitamina $D$ e de mineralização óssea em crianças e adolescentes residentes na cidade do Porto. Rev SPCNA. 2012;18:54.

19. Rocha A. Avaliação do estado de Vitamina $D$ numa população pediátrica do grande Porto. [Dissertação de Mestrado]. Porto: Instituto de Ciências Biomédicas Abel Salazar; 2012. [consultado 2015 jan 03]. Disponível em: http://hdl.handle.net/10216/62189.

20. Adams JS, Hewison M. Update in vitamin D. J Clin Endocrinol Metab. 2010;95:471-8.

21. Ashwell M, Stone EM, Stolte $H$, Cashman KD, Macdonald $H$, LanhamNew S, et al. UK Food Standards Agency workshop Report: an investigation of the relative contributions of diet and sunlight to vitamin D status. Br J Nutr. 2010;104:603-11. 


\section{Carência de Vitamina D numa População Hospitalar: Uma Fotografia pela Perspetiva Laboratorial}

Acta Med Port 2015:28:726-734

Publicado pela Acta Médica Portuguesa, a Revista Científica da Ordem dos Médicos

Av. Almirante Gago Coutinho, 151

1749-084 Lisboa, Portugal.

Tel: +351218428215

E-mail: submissao@actamedicaportuguesa.com

www.actamedicaportuguesa.com

ISSN:0870-399X | e-ISSN: 1646-0758

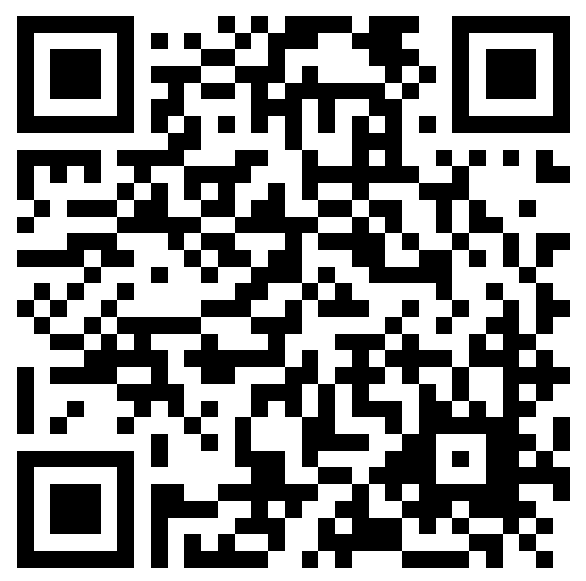

